\title{
Catastrophic Acute Thrombotic Occlusion During Fractional Flow Reserve Measurement
}

\author{
Arihide Okahara $^{\text {a }}$, Daigo Mine ${ }^{\mathrm{a}}$, Kenji Sadamatsua, b
}

\begin{abstract}
This report describes a patient presenting with thrombotic occlusion of the left circumflex artery as a complication associated with the measurement of the fractional flow reserve. When we measured the fractional flow reserve with a pressure wire through a 5-Fr diagnostic catheter to assess myocardial ischemia in the left anterior descending artery, the pressure wave showed a damping curve. Then, the patient collapsed, and a coronary angiogram showed thrombotic occlusion of the proximal circumflex artery. The thrombus was successfully retrieved by means of ballooning and thrombectomy, and thereafter the coronary flow recovered.
\end{abstract}

Keywords: Complication; Coronary intervention; Intravascular ultrasound; Thrombus

\section{Introduction}

Clinical studies support routine measurement of the fractional flow reserve (FFR) in patients with multivessel coronary artery disease undergoing percutaneous coronary intervention in order to reduce mortality and the rate of myocardial infarction [1]. In addition, recent guidelines recommend using the FFR to assess angiographic intermediate lesions in patients with a class IIa status [2]. Consequently, the number of patients assessed with the FFR is increasing, and physicians should thus be aware of the potential for a catastrophic complication during measurement of the FFR.

\section{Case Report}

A 76-year-old male who underwent balloon angioplasty of the

\footnotetext{
Manuscript accepted for publication April 29, 2015

${ }^{a}$ Department of Cardiology, Saga-ken Medical Centre Koseikan, Saga, Japan ${ }^{\mathrm{b} C}$ Corresponding Author: Kenji Sadamatsu, Department of Cardiology, Sagaken Medical Centre Koseikan, 400 Nakabaru, Kase, Saga 840-8571, Japan. Email: k-sadamatsu@umin.ac.jp
}

doi: http://dx.doi.org/10.14740/jmc2174w middle portion of the left anterior descending artery (LAD) 16 years previously was admitted to our hospital for treatment of effort angina. His coronary risk factors were hypertension and dyslipidemia. He was administered $100 \mathrm{mg}$ of aspirin per day. The results of an electrocardiogram and echocardiogram were normal. Thallium myocardial scintigraphy showed a small reversible perfusion defect in the LAD territory. A left coronary angiogram revealed intermediate stenotic lesions in the proximal left circumflex artery and proximal LAD (Fig. 1). A right coronary angiogram disclosed severe stenosis in the proximal right coronary artery.

We planned to evaluate the FFR in all three coronary arteries in order to select a treatment strategy. At the beginning of coronary angiography, 2,000 units of unfractionated heparin were administered. Following the calibration and equalization of the pressure wire, we inserted the wire into the distal LAD through a 5-Fr diagnostic catheter. Adenosine triphosphate was intravenously administered at a dose of $150 \mu \mathrm{g} / \mathrm{kg} / \mathrm{min}$ to induce hyperemia. The dose was subsequently increased to 180 $\mu \mathrm{g} / \mathrm{kg} / \mathrm{min}$; however, the FFR value was 0.91 (Fig. 2A). We pulled back the wire to the left main trunk and found that the FFR value was 1.3 with a damped waveform through the diagnostic catheter (Fig. 2B). Because aspirating and flushing the

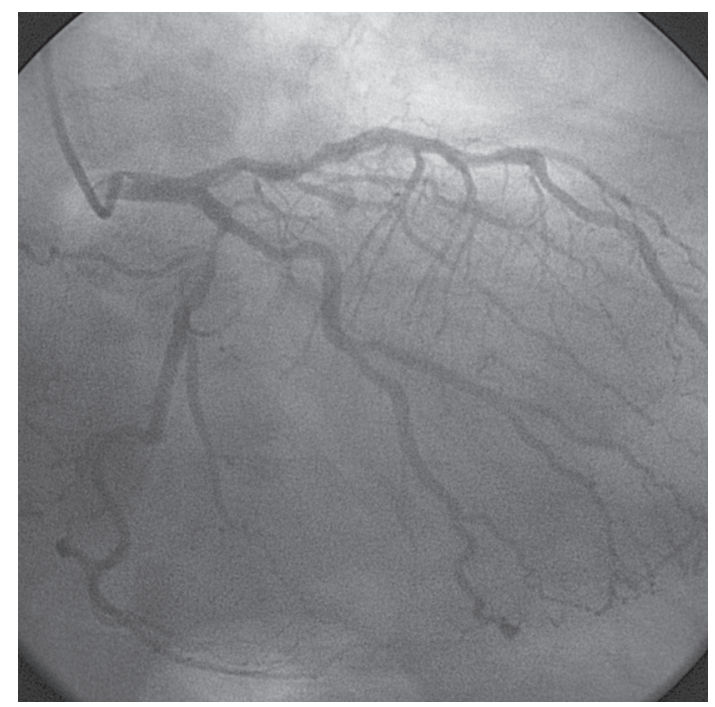

Figure 1. A left coronary angiogram showed intermediate lesions in the left anterior descending and circumflex arteries. 


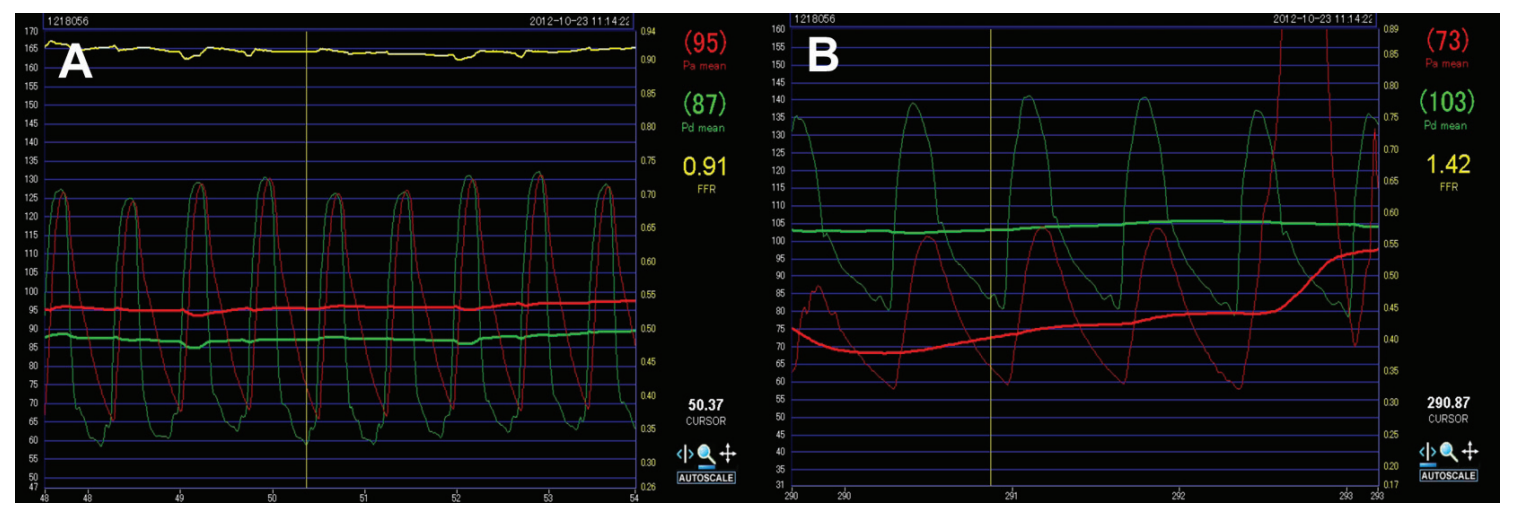

Figure 2. Measurement of the fractional flow reserve in the distal left anterior descending artery demonstrated a value of 0.91 (A). After measurement, we pulled back the pressure wire to the left main trunk, and the pressure curve (red line) obtained through the diagnostic catheter was damped (B).

catheter lumen did not improve the waveform, we exchanged the catheter for a new catheter. The patient then complained of chest pain with ST elevation in leads II, III, aVF and V5-6 and ST depression in leads I, aVL and V1-4. A left coronary angiogram revealed total occlusion in the proximal circumflex and obtuse marginal arteries (Fig. 3). The patient's blood pressure fell, and he became unconscious; therefore, we administered intravenous dopamine with an additional bolus injection of 5,000 units of unfractionated heparin. We placed a 6-Fr guiding catheter in the left coronary ostium and inserted a floppy wire into the circumflex artery. The intravenous ultrasound findings of the occluded lesion showed a thrombus without dissection or plaque rupture (Fig. 4). Balloon angioplasty with a $3.0 \times 15 \mathrm{~mm}$ balloon recovered the coronary flow. The obtuse marginal artery remained occluded, and we therefore inserted another floppy wire and dilated the lesion with the balloon. Because a coronary angiogram revealed distal embolization, we inserted a thrombectomy catheter and successfully retrieved a

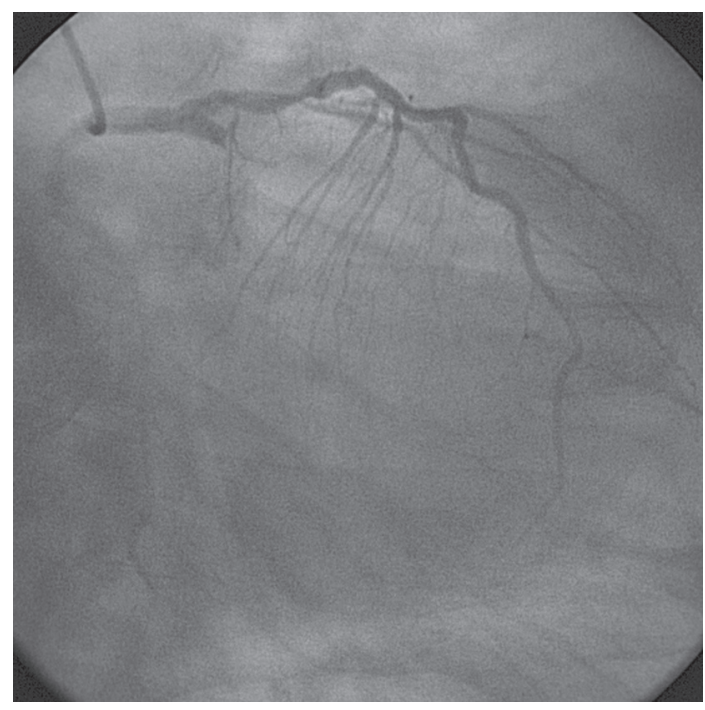

Figure 3. The left coronary angiogram obtained during the patient's sudden collapse revealed total occlusion in the proximal circumflex artery. large thrombus (Fig. 5). The final angiogram showed a good outcome, without residual narrowing or dissection.

\section{Discussion}

Measuring the FFR is a simple and easy method that involves using a coronary pressure wire to evaluate whether particular lesions are associated with myocardial ischemia. This procedure has been deemed safe, although manipulating the wire carries a risk of thrombus formation. In the present case, a thrombus formed in the diagnostic catheter during measurement of the FFR, which damped the waveform obtained through the catheter, then subsequently dropped into the left circumflex artery, resulting in occlusion. The administration of a low dose of heparin and use of a diagnostic catheter with a small lumen are important causative factors of thrombus formation in catheters. However, Legalery et al reported that FFR-related complications occurred in only one case of plaque dissection

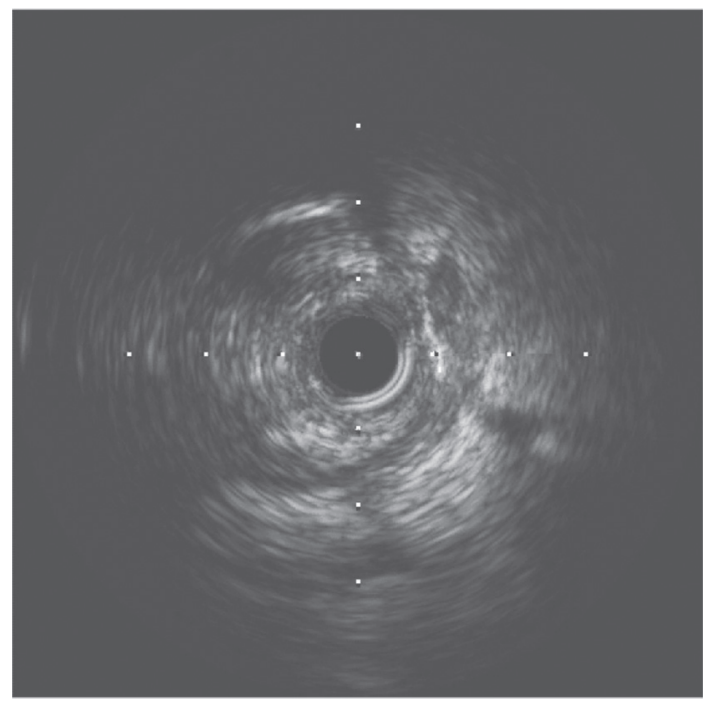

Figure 4. The intravenous ultrasound findings demonstrated thrombotic occlusion without dissection or plaque rupture. 


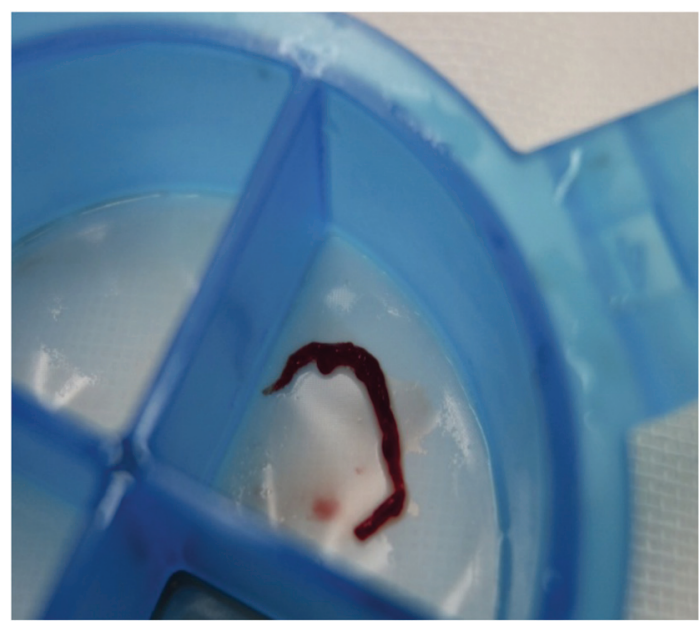

Figure 5. A large thrombus was successfully aspirated from the occluded circumflex artery using a thrombectomy catheter.

among 407 patients in whom the FFR was assessed using a 4- or 5-Fr diagnostic catheter in association with 25,000 units of heparin in $80 \%$ of cases [3], and therefore the incidence of thrombotic occlusion related to the measurement of the FFR is consisdered to be quite low.

\section{Conclusion}

Physicians should therefore take great care to prevent the occurrence of thrombotic complications when measuring the
FFR.

\section{Conflicts of Interest}

The authors report no financial relationships or conflicts of interest.

\section{References}

1. Pijls NH, Fearon WF, Tonino PA, Siebert U, Ikeno F, Bornschein B, van't Veer M, et al. Fractional flow reserve versus angiography for guiding percutaneous coronary intervention in patients with multivessel coronary artery disease: 2-year follow-up of the FAME (Fractional Flow Reserve Versus Angiography for Multivessel Evaluation) study. J Am Coll Cardiol. 2010;56(3):177-184.

2. Levine GN, Bates ER, Blankenship JC, Bailey SR, Bittl JA, Cercek B, Chambers CE, et al. 2011 ACCF/AHA/ SCAI Guideline for Percutaneous Coronary Intervention: a report of the American College of Cardiology Foundation/American Heart Association Task Force on Practice Guidelines and the Society for Cardiovascular Angiography and Interventions. Circulation. 2011;124(23):e574651 .

3. Legalery P, Schiele F, Seronde MF, Meneveau N, Wei $\mathrm{H}$, Didier K, Blonde MC, et al. One-year outcome of patients submitted to routine fractional flow reserve assessment to determine the need for angioplasty. Eur Heart J. 2005;26(24):2623-2629. 